\title{
Synthesis of $N^{\alpha}$-protected aminoacid/peptide Weinreb amides employing $N, N^{\prime}$-carbonyldiimidazole as activating agent; studies on docking and antibacterial activities
}

\author{
K. Uma, ${ }^{a}$ H.S. Lalithamba, ${ }^{* a}$ M. Raghavendra, ${ }^{a}$ Vivek Chandramohan, ${ }^{b}$ and C. Anupama ${ }^{b}$ \\ ${ }^{a}$ Department of Chemistry, Siddaganga Institute of Technology, B.H. Road, \\ Tumakuru-572 103, Karnataka, India \\ ${ }^{b}$ Department of Biotechnology, Siddaganga Institute of Technology, B.H. Road, \\ Tumakuru-572 103, Karnataka, India \\ Email:lalithambasit@yahoo.co.in
}

DOI: http://dx.doi.org/10.3998/ark.5550190.p009.605

\begin{abstract}
An efficient method for the synthesis of $N^{\alpha}$-protected amino/ peptide Weinreb amides methoxy- $N$-methylamides) employing $N, N^{\prime}$-carbonyldiimidazole (CDI) has been achieved. $N^{\alpha}$ protected amino/peptide acids were treated with $N, N^{\prime}$-carbonyldiimidazole, followed by the addition of $\mathrm{N}, \mathrm{O}$-dimethylhydroxylamine hydrochloride salt to yield the desired compounds. The synthesized compounds were mainly gums, a few were solids, after the simple workup, and were characterized by IR, ${ }^{1} \mathrm{H}$ NMR, ${ }^{13} \mathrm{C}$ NMR and HRMS. The Weinreb amides were subjected to in silico studies, to predict the preferred orientation and binding affinity between the molecules using scoring functions. The ligand $\mathrm{N}$-Fmoc-L-Phe-N $\left(\mathrm{OCH}_{3}\right) \mathrm{CH}_{3}$ showed minimum binding energy $29.85 \mathrm{kcal} / \mathrm{mol}$ with Escherichia coli and the ligand $N$-Fmoc-L-Ala- $\mathrm{N}\left(\mathrm{OCH}_{3}\right) \mathrm{CH}_{3}$ showed minimum binding energy $-24.79 \mathrm{kcal} / \mathrm{mol}$ with Pseudomonas aeruginosa, $-25.01 \mathrm{kcal} / \mathrm{mol}$ with Staphylococcus aureus. Based on the minimum binding energies, antibacterial activities have been conducted for a few of the synthesized compounds.
\end{abstract}

Key words: Weinreb amides, $N, O$-dimethylhydroxylamine hydrochloride, $N, N^{\prime}$-carbonyldiimidazole, in silico molecular docking studies, in vitro antibacterial activities

\section{Introduction}

Weinreb amides have wide importance in organic synthesis due to their versatile reactivity with nucleophiles and selective reduction to aldehydes. Weinreb amides derived from amino acids have been extensively used as precursors in the preparation of $\alpha$-amino aldehydes and $\alpha$-amino ketones. They are selectively reduced to aldehydes using $\mathrm{LiAlH}_{4}$ and form ketones upon reaction with 
Grignard reagents. These $\alpha$-amino ketones are the intermediates in the synthesis of many drugs and biologically active compounds. ${ }^{1,2,8}$ They are also useful in the synthesis of acetylenes which are starting materials for the popular click reactions.

The reported protocols to convert carboxylic acids into the corresponding Weinreb amides follow the method of activation of the carboxylic group mainly via mixed anhydride using chloroformates followed by coupling with $\mathrm{N}, \mathrm{O}$-dimethylhydroxylamine. Quite a few common peptide coupling reagents such as BOP, DCC, CDMT, DMTMM, COMU etc., are alternatives for this transformation. ${ }^{3,12}$ One-pot synthesis of $N$-Boc $\alpha$-amino Weinreb amides using acid fluorides as key intermediates employing [bis-(2-methoxyethyl)amino]sulfur trifluoride (Deoxo-fluor) has also been discussed. ${ }^{4,5}$ Sureshbabu et al. synthesized Weinreb amides employing acid chlorides as key intermediates. Further the use of carboxylic acid-activating reagents like $N, N^{\prime}$ dicyclohexylcarbodiimide, propylphosphonic anhydride/ $N$-ethylmorpholine, $N$-benzotriazole derivatives, $S, S^{\prime}$-di-(2-pyridyl)dithiocarbonate, and 2-bromo-1-methylpyridinium iodide are reported. ${ }^{6}$ Palladium-catalyzed preparation of Weinreb amides from boronic acids is described. ${ }^{7}$ Deagostino and co-workers developed a protocol for the synthesis of Weinreb amides via Pdcatalyzed aminocarbonylation of heterocyclic-derived triflates. ${ }^{9}$ The combination of $\mathrm{PPh}_{3} / \mathrm{I}_{2}$ and T3P/DBU has proved to be effective for conversion of $N^{\alpha}$-protected amino acids into the corresponding Weinreb amides. ${ }^{10,11}$ However, many of the procedures mentioned are unattractive due to disadvantages such as longer reaction times, low yields, multi-step reactions, etc.

In this paper we report the activation of carboxylic acids with $N, N^{\prime}$-carbonyldiimidazole (CDI). Carbonyl diimidazole is a useful coupling reagent that promotes peptide bond formation and is also used for the preparation of ureas and carbamates from amines and alcohols respectively. ${ }^{13}$ Aspirin prodrugs in one pot were synthesized employing CDI. ${ }^{14}$ This reagent is commonly used on a large scale in peptide chemistry and its use can be extended to the formation of esters and thioesters. ${ }^{15}$ The reaction of secondary amines using $N, N^{\prime}$-carbonyldiimidazole, was described in the literature for the synthesis of tertiary amides. ${ }^{16}$ Synthesis of $N^{\alpha}$-protected amino acid azides employing CDI and its application for the preparation of ureidopeptides was investigated. ${ }^{17}$ Azolide ${ }^{18}$ and oxadiazolone synthesis in good yield by treatment of the hydrazide with CDI in refluxing dioxane was reported. ${ }^{19}$ Aromatic amides and esters have been synthesized by in situ activation of hydroxy acids using CDI mediated coupling. ${ }^{20} N, N^{\prime}$ - carbonyldiimidazole is one of several universally used reagents for the activation carboxyl groups. It is relatively cheap and the byproducts are carbon dioxide and imidazole. Therefore, an efficient method for the synthesis of $N^{\alpha}$-protected-amino-peptidyl Weinreb amides is desirable. Herein, we report an efficient, one pot synthesis of $N^{\alpha}$-protected amino acid/peptide acid-derived Weinreb amides employing CDI as activating agent. The prepared compounds were screened for in silico molecular docking studies and in vitro antibacterial activities. Docking aims to predict accurately the structure of a ligand within the constraints of a receptor binding site and correctly to estimate the strength of binding between the molecules. ${ }^{21,22}$ 


\section{Results and Discussion}

Chemistry: For the synthesis of the $N^{\alpha}$-protected Weinreb amides (2a-2m), $N^{\alpha}$-protected amino acid was dissolved in THF; CDI was added at $0^{\circ} \mathrm{C}$ and the solution was stirred for about $10 \mathrm{~min}$. Then, $N, O$-dimethylhydroxylamine hydrochloride salt in dry DCM neutralized by the addition of $N$-methylmorpholine (NMM) was added. The reaction mixture was stirred till the completion of the reaction as indicated by TLC. After simple work up, the desired products were obtained in good yield (Scheme 1). In this way several Weinreb amides were synthesized from $N^{\alpha}$-protected Fmoc/Cbz/Boc amino acids (Table 1).

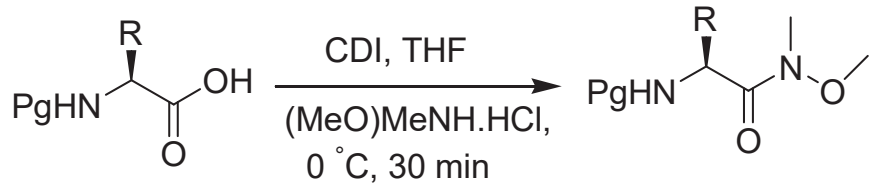

1

$2 a-2 m$

$\mathrm{Pg}=\mathrm{Fmoc} / \mathrm{Boc}$ or $\mathrm{Cbz}$ protecting group $\mathrm{R}=$ Amino acid side chains

Scheme 1. Synthesis of $N^{\alpha}$-protected Weinreb amides.

Table 1. List of amino acid Weinreb amides prepared following Scheme 1

\begin{tabular}{cccc}
\hline Compound & Weinreb amides & Yield (\%) & M.p. $/{ }^{\circ} \mathrm{C}$ \\
\hline $\mathbf{2 a}$ & $N$-Fmoc-L-Phe-N $\left(\mathrm{OCH}_{3}\right) \mathrm{CH}_{3}$ & 89 & 130 \\
$\mathbf{2 b}$ & $N$-Fmoc-L-Ala-N $\left(\mathrm{OCH}_{3}\right) \mathrm{CH}_{3}$ & 85 & 114 \\
$\mathbf{2 c}$ & $N$-Fmoc-L-Val-N $\left(\mathrm{OCH}_{3}\right) \mathrm{CH}_{3}$ & 83 & 109 \\
$\mathbf{2 d}$ & $N$-Fmoc-L-Leu-N $\left(\mathrm{OCH}_{3}\right) \mathrm{CH}_{3}$ & 82 & 125 \\
$\mathbf{2 e}$ & $N$-Fmoc-L-Trp-N $\left(\mathrm{OCH}_{3}\right) \mathrm{CH}_{3}$ & 87 & Gum \\
$\mathbf{2 f}$ & $N$-Fmoc-L-Pro-N $\left(\mathrm{OCH}_{3}\right) \mathrm{CH}_{3}$ & 90 & Gum \\
$\mathbf{2 g}$ & $N$-Cbz -L-Ala-N $\left(\mathrm{OCH}_{3}\right) \mathrm{CH}_{3}$ & 86 & 110 \\
$\mathbf{2 h}$ & $N$-Cbz -L-Ser-N $\left(\mathrm{OCH}_{3}\right) \mathrm{CH}_{3}$ & 80 & Gum \\
$\mathbf{2 i}$ & $N$-Cbz -L-Gly-N $\left(\mathrm{OCH}_{3}\right) \mathrm{CH}_{3}$ & 88 & Gum \\
$\mathbf{2 j}$ & $N$-Boc-L-Phe-N $\left(\mathrm{OCH}_{3}\right) \mathrm{CH}_{3}$ & 90 & 106 \\
$\mathbf{2 k}$ & $N$-Boc-L-Met-N $\left(\mathrm{OCH}_{3}\right) \mathrm{CH}_{3}$ & 81 & Gum \\
$\mathbf{2 l}$ & $N$-Boc-L-Ile-N $\left(\mathrm{OCH}_{3}\right) \mathrm{CH}_{3}$ & 80 & Gum \\
$\mathbf{2 m}$ & $N$-Boc-L-Leu-N $\left(\mathrm{OCH}_{3}\right) \mathrm{CH}_{3}$ & 86 & Gum \\
\hline
\end{tabular}


$N^{\alpha}$-protected peptidyl Weinreb amides (Table 2, 4n-4p) were also prepared starting from Fmoc-peptide acids (Scheme 2). Chiral HPLC analysis was carried out for the enantiomeric pair of Fmoc-L-Ala- and -D-Ala-Weinreb amide and the D, L mixture. The samples showed distinct peak with retention times at $10.8 \mathrm{~min}$ and $14.6 \mathrm{~min}$ respectively. The equimolar mixture of $\mathrm{D}-$ and L-enantiomers under similar conditions showed a significant difference in the retention times between D- and L-amino acid derivatives with retention times 11.0 and $14.3 \mathrm{~min}$.<smiles>[R]C(NC(=O)O)C(=O)NC([R])C(=O)O[Na]</smiles>

3<smiles>[R]C(NC(=O)O)C(=O)NC([R])C(=O)N(C)C</smiles>

$4 n-4 p$

$\mathrm{Pg}=$ Fmoc/Boc or Cbz group; $\mathrm{R}^{1}, \mathrm{R}^{2}=$ Amino acid side chains

Scheme 2. Synthesis of $N^{\alpha}$-protected peptidyl Weinreb amides.

Table 2. Peptidyl Weinreb amides prepared following Scheme 2

\begin{tabular}{cccc}
\hline Compound & Peptidyl Weinreb amides & Yield (\%) & M.p./ ${ }^{\circ} \mathrm{C}$ \\
\hline $\mathbf{4 n}$ & $N$-Fmoc-L-Gly-Pro-N $\left(\mathrm{OCH}_{3}\right) \mathrm{CH}_{3}$ & 90 & Gum \\
$\mathbf{4 0}$ & $N$-Fmoc-L-Arg-Ala-N $\left(\mathrm{OCH}_{3}\right) \mathrm{CH}_{3}$ & 84 & Gum \\
$\mathbf{4 p}$ & $N$-Fmoc-L-Arg-Gly-N $\left(\mathrm{OCH}_{3}\right) \mathrm{CH}_{3}$ & 88 & Gum \\
\hline
\end{tabular}

The docking results for top five compounds against all the three different receptor proteins are tabulated in table 3 .

Table 3(a). Docking results of Weinreb amides with Escherichia coli

\begin{tabular}{clc}
\hline Entry & Compound name & $\begin{array}{c}\text { Escherichia coli } \\
\text { Docking score }\end{array}$ \\
\hline 1. & Fmoc-L-Phe-N $\left(\mathrm{OCH}_{3}\right) \mathrm{CH}_{3}$ & -29.85 \\
2. & Fmoc-L-Ala-N $\left(\mathrm{OCH}_{3}\right) \mathrm{CH}_{3}$ & -27.46 \\
3. & Fmoc-L-Arg-N(OCH$) \mathrm{CH}_{3}$ & -26.06 \\
4. & Fmoc-L-Asn-N $\left(\mathrm{OCH}_{3}\right) \mathrm{CH}_{3}$ & -25.61 \\
5. & Fmoc-L-Cys-N(OCH$) \mathrm{CH}_{3}$ & -24.21 \\
6. & Fmoc-L-Ser-N $\left(\mathrm{OCH}_{3}\right) \mathrm{CH}_{3}$ & -24.01 \\
\hline
\end{tabular}


Table 3(b). Docking results of Weinreb amides with Pseudomonas aeroginosa

\begin{tabular}{ccc}
\hline Entry & Compound name & $\begin{array}{c}\text { Pseudomonas aeroginosa } \\
\text { Docking score }\end{array}$ \\
\hline 1 & Fmoc-L-Ala-N $\left(\mathrm{OCH}_{3}\right) \mathrm{CH}_{3}$ & -24.79 \\
2 & Fmoc-L-Phe-N $\left(\mathrm{OCH}_{3}\right) \mathrm{CH}_{3}$ & -23.63 \\
3 & $\mathrm{~L}-\mathrm{Glu}-\mathrm{N}\left(\mathrm{OCH}_{3}\right) \mathrm{CH}_{3}$ & -21.34 \\
4 & Fmoc-L-Gln-N($\left(\mathrm{OCH}_{3}\right) \mathrm{CH}_{3}$ & -21.08 \\
5 & $\mathrm{~L}-\mathrm{Arg}-\mathrm{N}\left(\mathrm{OCH}_{3}\right) \mathrm{CH}_{3}$ & -20.20 \\
6 & $\mathrm{~L}-\mathrm{Gln}-\mathrm{N}\left(\mathrm{OCH}_{3}\right) \mathrm{CH}_{3}$ & -19.65 \\
\hline
\end{tabular}

Table 3(c). Docking results of Weinreb Amides with Staphylococcus aureus

\begin{tabular}{ccc}
\hline Entry & Compound name & $\begin{array}{c}\text { Staphylococcus aureus } \\
\text { Docking score }\end{array}$ \\
\hline 1 & Fmoc-L-Ala-N $\left(\mathrm{OCH}_{3}\right) \mathrm{CH}_{3}$ & -25.01 \\
2 & Fmoc-L-Phe-N $\left(\mathrm{OCH}_{3}\right) \mathrm{CH}_{3}$ & -21.60 \\
3 & Fmoc-L-Arg- $\left(\mathrm{OCH}_{3}\right) \mathrm{CH}_{3}$ & -13.28 \\
4 & L-His-N $\left(\mathrm{OCH}_{3}\right) \mathrm{CH}_{3}$ & -10.08 \\
5 & L-Pro-N $\left(\mathrm{OCH}_{3}\right) \mathrm{CH}_{3}$ & -9.13 \\
6 & Fmoc-L-Trp- $\left(\mathrm{OCH}_{3}\right) \mathrm{CH}_{3}$ & -8.98 \\
\hline
\end{tabular}

Figure 1 shows the pictorial representation of the binding modes of synthesized molecules with target proteins. The ligand $\mathrm{N}$-Fmoc-L-Phe- $\mathrm{N}\left(\mathrm{OCH}_{3}\right) \mathrm{CH}_{3}$ showed minimum binding energy -29.85 $\mathrm{kcal} / \mathrm{mol}$ with Escherichia coli and the ligand $N$-Fmoc-L-Ala- $\mathrm{N}\left(\mathrm{OCH}_{3}\right) \mathrm{CH}_{3}$ showed minimum binding energy $-24.79 \mathrm{kcal} / \mathrm{mol}$ with Pseudomonas aeruginosa, $-25.01 \mathrm{kcal} / \mathrm{mol}$ with Staphylococcus aureus. In silico results indicate, $N$-Fmoc-L-Phe-N $\left(\mathrm{OCH}_{3}\right) \mathrm{CH}_{3}$ and $N$-Fmoc-LAla- $\mathrm{N}\left(\mathrm{OCH}_{3}\right) \mathrm{CH}_{3}$ were showing least binding energy. These lead molecules were evaluated in in vitro studies.

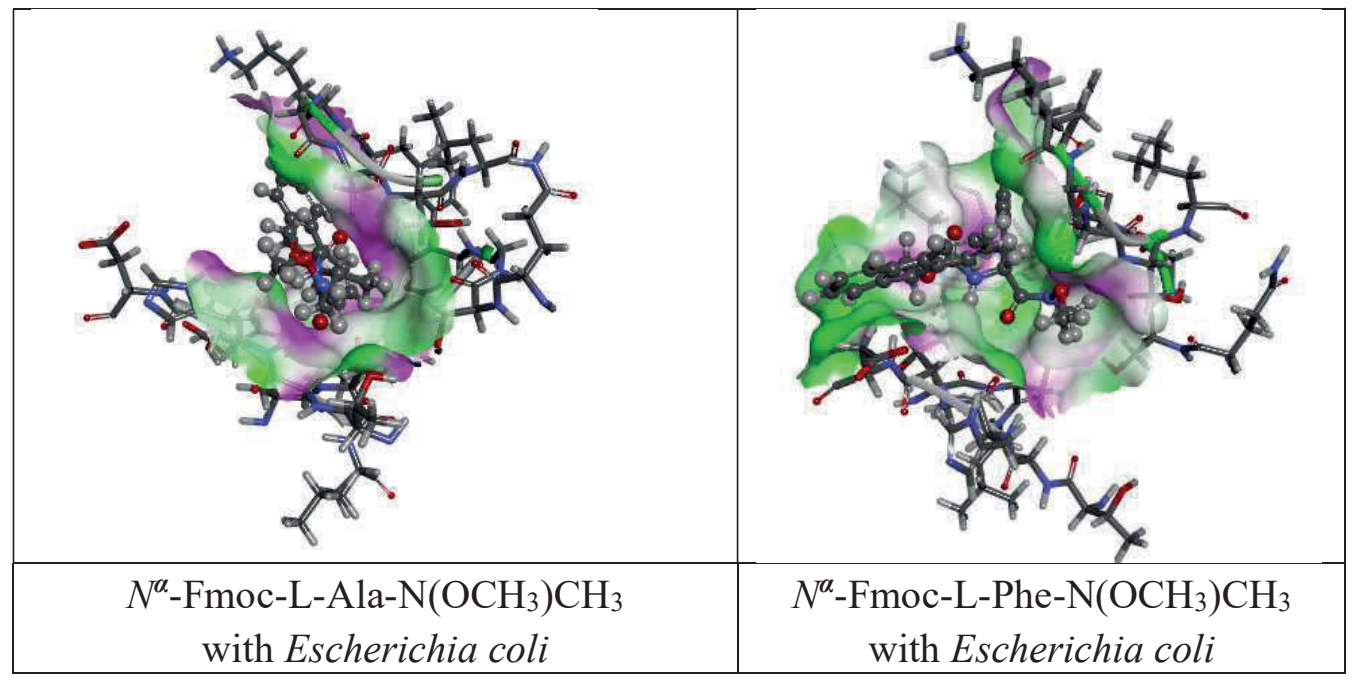




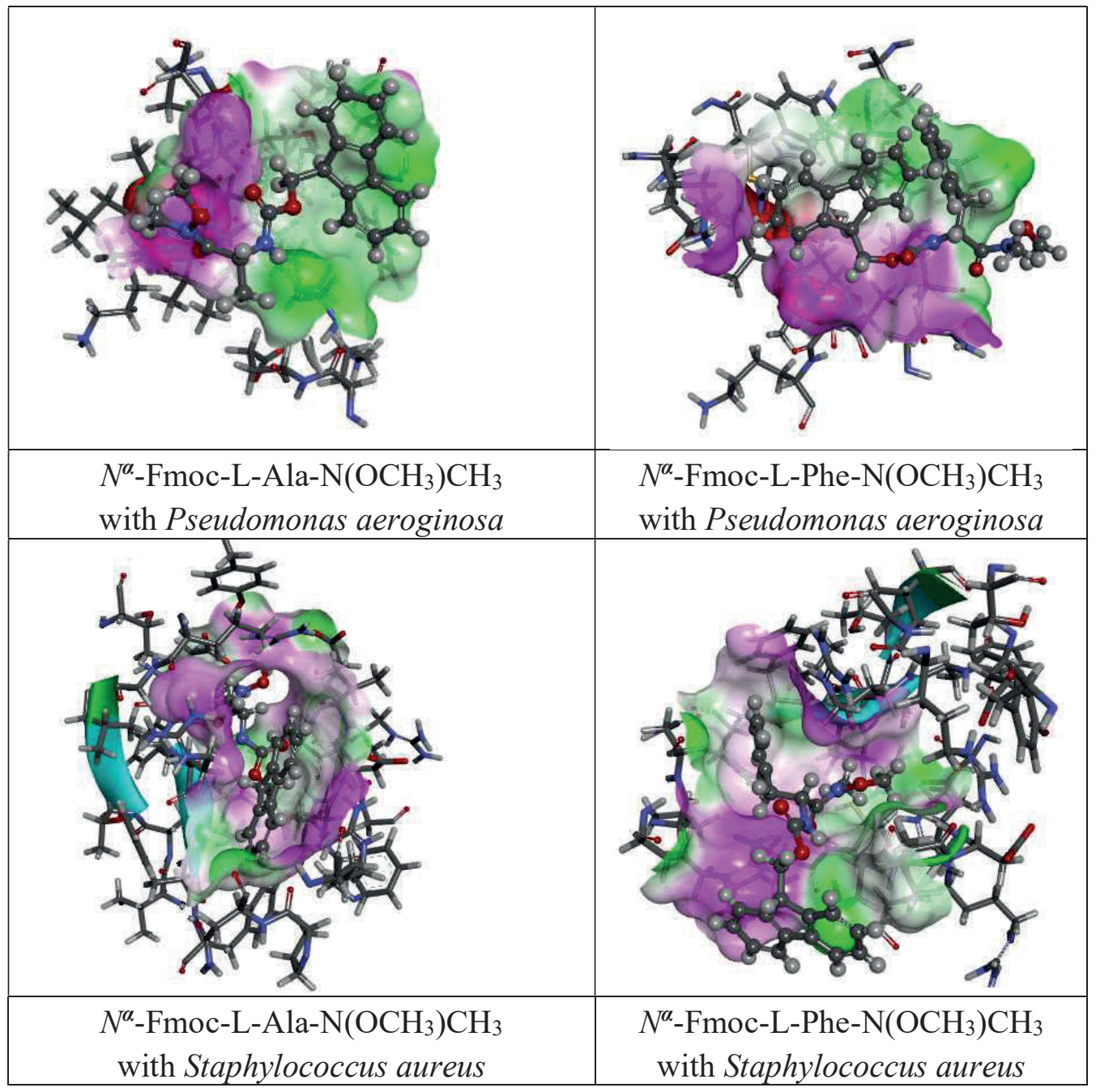

Figure 1. Pictorial representations of the binding modes of synthesized molecules with target proteins.

The antibacterial results of Escherichia coli, Pseudomonas aeruginosa, and Staphylococcus aureus are tabulated in Table 4. Based on the docking results the $\mathrm{N}$-Fmoc-L-Ala- $\mathrm{N}\left(\mathrm{OCH}_{3}\right) \mathrm{CH}_{3}$ and $N$-Fmoc-L-Phe- $\mathrm{N}\left(\mathrm{OCH}_{3}\right) \mathrm{CH}_{3}$ were showing good activity in in vitro studies. The compound $N$-Fmoc-L-Phe- $\mathrm{N}\left(\mathrm{OCH}_{3}\right) \mathrm{CH}_{3}$ showed inhibition zone of $14 \mathrm{~mm}$ against Escherichia coli, $N$-FmocL-Ala-N $\left(\mathrm{OCH}_{3}\right) \mathrm{CH}_{3}$ showed inhibition zone of $14 \mathrm{~mm}$ against Pseudomonas aeruginosa and $\mathrm{N}$ Fmoc-L-Ala-N $\left(\mathrm{OCH}_{3}\right) \mathrm{CH}_{3}$ showed inhibition zone of $14 \mathrm{~mm}$ against Staphylococcus aureus compared to standard compound. 
Table 4. Antibacterial test for protected Weinreb amides

\begin{tabular}{cccccc}
\hline Entry & Sample name & Standard & \multicolumn{3}{c}{ Sample concentration } \\
\hline & Escherichia coli & & $\mathbf{5 0}$ & $\mathbf{1 0 0}$ & $\mathbf{2 0 0}$ \\
1. & $N$-Fmoc-L-Ala-N $\left(\mathrm{OCH}_{3}\right) \mathrm{CH}_{3}$ & $14 \mathrm{~mm}$ & $10 \mathrm{~mm}$ & $12 \mathrm{~mm}$ & $12 \mathrm{~mm}$ \\
2. & $N$-Fmoc-L-Phe-N $\left(\mathrm{OCH}_{3}\right) \mathrm{CH}_{3}$ & $14 \mathrm{~mm}$ & $14 \mathrm{~mm}$ & $10 \mathrm{~mm}$ & $12 \mathrm{~mm}$ \\
& Pseudomonas aeruginosa & & $\mathbf{5 0}$ & $\mathbf{1 0 0}$ & $\mathbf{2 0 0}$ \\
1. & $N$-Fmoc-L-Ala- $\left(\mathrm{OCH}_{3}\right) \mathrm{CH}_{3}$ & $14 \mathrm{~mm}$ & $14 \mathrm{~mm}$ & $13 \mathrm{~mm}$ & $13 \mathrm{~mm}$ \\
2. & $N$-Fmoc-L-Phe- $\left(\mathrm{OCH}_{3}\right) \mathrm{CH}_{3}$ & $14 \mathrm{~mm}$ & $8 \mathrm{~mm}$ & $10 \mathrm{~mm}$ & $10 \mathrm{~mm}$ \\
& $\quad$ Staphylococcus aureus & & $\mathbf{5 0}$ & $\mathbf{1 0 0}$ & $\mathbf{2 0 0}$ \\
1. & $N$-Fmoc-L-Ala- $\left(\mathrm{OCH}_{3}\right) \mathrm{CH}_{3}$ & $13 \mathrm{~mm}$ & $14 \mathrm{~mm}$ & $8 \mathrm{~mm}$ & $10 \mathrm{~mm}$ \\
2. & $N$-Fmoc-L-Phe-N $\left(\mathrm{OCH}_{3}\right) \mathrm{CH}_{3}$ & $13 \mathrm{~mm}$ & $9 \mathrm{~mm}$ & $11 \mathrm{~mm}$ & $10 \mathrm{~mm}$ \\
\hline
\end{tabular}

\section{Conclusions}

In the present work, we have used Fmoc/Cbz/Boc $\alpha$-amino/peptide acids as precursors for the preparation of Fmoc/Cbz/Boc $\alpha$-amino/peptidyl Weinreb amides. The carboxylic group of amino acids was activated using CDI followed by the coupling reaction with $N, O$ - dimethyl hydroxylamine hydrochloride salt to obtain the title products. This protocol is an efficient method for the synthesis of Weinreb amides. All the products were isolated after simple work up and were fully characterized by IR, ${ }^{1} \mathrm{H}$ NMR, ${ }^{13} \mathrm{C}$ NMR and mass spectroscopy. Finally, the synthesized products were subjected to molecular docking studies and antibacterial activities employing Escherichia coli, Pseudomonas aeroginosa and Staphylococcus aureus. The lead compound, $N$ Fmoc-L-Phe-N $\left(\mathrm{OCH}_{3}\right) \mathrm{CH}_{3}$ showed least binding energy of $-29.85 \mathrm{kcal} / \mathrm{mol}$ with enoyl-ACP reductase (1C14) Escherichia coli, the lead compound $N$-Fmoc-L-Ala- $\mathrm{N}\left(\mathrm{OCH}_{3}\right) \mathrm{CH}_{3}$ showed least binding energy of $-24.79 \mathrm{kcal} / \mathrm{mol}$ with LasR ligand binding domain bound to its natural ligand $n$ 3-oxododecanoyl-L-homoserine lactone (2UV0) Pseudomonas aeroginosa and the lead compound $\mathrm{N}$-Fmoc-L-Ala-N $\left(\mathrm{OCH}_{3}\right) \mathrm{CH}_{3}$ showed least binding energy $-25.01 \mathrm{kcal} / \mathrm{mol}$ with dehydrosqualene synthase (2ZCP) Staphylococcus aureus.

\section{Experimental Section}

General. All chemicals were purchased from Sigma-Aldrich and Merck and used without purification. The solvents were freshly distilled before use. Melting points were taken in open capillaries. TLC analysis was carried out using precoated silica gel $F_{254}$. IR spectra were recorded on Agilent Cary 620 FT-IR spectrometer. ${ }^{1} \mathrm{H}$ NMR spectra were done on a Bruker AMX $400 \mathrm{MHz}$ spectrometer using $\mathrm{Me}_{4} \mathrm{Si}$ as an internal standard and $\mathrm{CDCl}_{3}$ as a solvent. Mass spectra were recorded on a Micromass Q-ToF Micro Mass Spectrometer. Acronyms in this section are defined 
as follows: CDI carbonyldiimidazole; THF tetrahydrofuran; DCM dichloromethane; NMM Nmethylmorpholine; Boc, Fmoc, $\mathrm{Cbz}$ - as defined in the compound names.

General procedure for the synthesis of $N^{\alpha}$ - protected amino/peptidyl Weinreb amides. To a stirred solution of protected amino/peptide acid (1 mmol) in THF, NMM (1.5 mmol) and CDI (1.5 mmol) was added at $0{ }^{\circ} \mathrm{C}$, followed by the addition of $\mathrm{N}, \mathrm{O}$-dimethylhydroxylamine hydrochloride $(1.1 \mathrm{mmol})$ in dry DCM (5-6 mL), neutralized with NMM. The reaction mixture was stirred till the completion of reaction. THF was removed and the product was extracted into ethyl acetate and the organic layer was washed with hydrochloric acid solution $(10 \mathrm{~mL})$ or citric acid solution (in case of Boc-protected compounds), sodium carbonate solution $(15 \mathrm{~mL} \times 2)$, water $(15 \mathrm{~mL})$ and brine $(15 \mathrm{~mL})$. It was dried over anhydrous sodium sulfate and concentrated.

\section{Physical and spectral data of the synthesized compounds}

$N^{\alpha}$-Fmoc-Phe-N $\left(\mathrm{OCH}_{3}\right) \mathrm{CH}_{3}$ (2a). ( $(S)$-(9H-Fluoren-9-yl)methyl-1-(methoxy(methyl)amino)1-oxo-3-phenylpropan-2-ylcarbamate. Yield $89 \%$, mp $130{ }^{\circ} \mathrm{C}$. $\mathrm{R}_{f}$ (ethyl acetate/ $n$-hexane 2:8) 0.6, IR (KBr, cm $\left.{ }^{-1}\right): 1660 ;{ }^{1} \mathrm{H}$ NMR (400 MHz, $\left.\mathrm{CDCl}_{3}\right): \delta(\mathrm{ppm}) 1.59(\mathrm{~s}, 3 \mathrm{H}), 2.91-3.12(\mathrm{~m}, 2 \mathrm{H})$, $3.65(\mathrm{~s}, 3 \mathrm{H}), 4.16-4.19(\mathrm{t}, J 8.0 \mathrm{~Hz}, 1 \mathrm{H}), 4.24-4.39(\mathrm{~m}, 2 \mathrm{H}), 5.00-5.05(\mathrm{~m}, 1 \mathrm{H}), 5.48-5.50(\mathrm{~d}, J 8.0$ $\mathrm{Hz}, 1 \mathrm{H}), 7.15-7.76(\mathrm{~m}, 13 \mathrm{H}) .{ }^{13} \mathrm{C} \mathrm{NMR}\left(100 \mathrm{MHz}, \mathrm{CDCl}_{3}\right): \delta$ (ppm) 32.05, 38.65, 47.10, 52.04, 61.52 , 66.95, 125.09, 125.15, 126.88, 127.00, 127.62, 128.38, 129.41, 136.26, 141.23, 143.85, 155.73, 171.87. MS: Calc. for $\mathrm{C}_{26} \mathrm{H}_{26} \mathrm{~N}_{2} \mathrm{O}_{4}: m / z 453.1790\left(\mathrm{M}^{+}+\mathrm{Na}\right)$, found: 453.1793 .

$\mathrm{N}^{a}$-Fmoc-Ala-N(OCH 3$) \mathrm{CH}_{3}(2 \mathrm{~b})$. ( $\mathrm{S}$ )-(9H-fluoren-9-yl)methyl-1-(methoxy(methyl)amino)-1oxopropan-2-ylcarbamate. Yield $85 \%, \mathrm{mp} 114^{\circ} \mathrm{C}$. $\mathrm{R}_{f}$ (ethyl acetate $/ n$ - hexane $2: 8$ ) 0.4 , IR (KBr, $\mathrm{cm}^{-1}$ ): 1635. ${ }^{1} \mathrm{H}$ NMR (400 MHz, $\left.\mathrm{CDCl}_{3}\right): \delta(\mathrm{ppm}) 1.36-1.38$ (d, $\left.J 8.0 \mathrm{~Hz}, 3 \mathrm{H}\right), 3.22(\mathrm{~s}, 3 \mathrm{H}), 3.77$ (s, 3H), 4.20-4.24 (t, J 8.0 Hz, 1H), 4.35-4.37 (d, J 8.0 Hz, 2H), 4.74-4.77 (t, J 4.0 Hz, 1H), 5.57$5.61(\mathrm{~d}, J 8.0 \mathrm{~Hz}, 1 \mathrm{H}), 7.29-7.77(\mathrm{~m}, 8 \mathrm{H}) .{ }^{13} \mathrm{C} \mathrm{NMR}\left(100 \mathrm{MHz}, \mathrm{CDCl}_{3}\right): \delta(\mathrm{ppm}) 18.65,32.14$, 47.11, 47.15, 61.59 (59, 66.93, 125.12, 125.15, 127.02, 127.64, 141.25, 143.83, 143.95, 155.72. MS: Calc. for $\mathrm{C}_{20} \mathrm{H}_{22} \mathrm{~N}_{2} \mathrm{O}_{4}: m / z 377.1477\left(\mathrm{M}^{+}+\mathrm{Na}\right)$, found: 377.1479 .

$\mathrm{N}^{\alpha}$-Fmoc-Val-N(OCH$) \mathrm{CH}_{3}$ (2c). (S)-(9H-fluoren-9-yl)methyl-1-(methoxy(methyl)amino)-3methyl-1-oxobutan-2-ylcarbamate. Yield $83 \%, \mathrm{mp} 109^{\circ} \mathrm{C}$. $\mathrm{R}_{f}$ (ethyl acetate/ $n$-hexane 2:8) 0.55 , IR (KBr, cm ${ }^{-1}$ ): $1654 .{ }^{1} \mathrm{H}$ NMR (400 MHz, $\left.\mathrm{CDCl}_{3}\right): \delta(\mathrm{ppm})$ 0.86-0.98 (m, 6H), 1.98-2.07 (m, 1H), $3.22(\mathrm{~s}, 3 \mathrm{H}), 3.77(\mathrm{~s}, 3 \mathrm{H}), 4.20-4.23(\mathrm{t}, J 8.0 \mathrm{~Hz}, 1 \mathrm{H}), 4.30-4.42(\mathrm{~m}, 1 \mathrm{H}), 4.62-4.66(\mathrm{~d}, J 8.0 \mathrm{~Hz}$, $1 \mathrm{H}), 5.46-5.48(\mathrm{~d}, J 8.0 \mathrm{~Hz}, 1 \mathrm{H}), 7.25-7.64(\mathrm{~m}, 8 \mathrm{H}) .{ }^{13} \mathrm{C} \mathrm{NMR}\left(100 \mathrm{MHz}, \mathrm{CDCl}_{3}\right): \delta(\mathrm{ppm}) 17.61$, 29.63, 31.86, 47.16, 55.55, 61.51, 68.73, 119.88, 124.37, 126.99, 129.22, 141.24, 143.81, 156.41, 172.56. MS: Calc. for $\mathrm{C}_{22} \mathrm{H}_{26} \mathrm{~N}_{2} \mathrm{O}_{4} \mathrm{~m} / z$ : $405.1900\left(\mathrm{M}^{+}+\mathrm{Na}\right)$, found: 405.1901.

\section{$N^{\alpha}$-Fmoc-L-Leu-N(OCH $)_{3} \mathrm{CH}_{3} \quad$ (2d). $\quad(S)$-(9H-fluoren-9-yl)methyl-1-(methoxy-} (methyl)amino)-4-methyl-1-oxopentan-2-ylcarbamate. Yield $82 \%, \mathrm{mp} 125{ }^{\circ} \mathrm{C}, \mathrm{R}_{f}$ (ethyl acetate/ $n$ - hexane $2: 8)=0.58, \mathrm{IR}\left(\mathrm{KBr}, \mathrm{cm}^{-1}\right): 1645 .{ }^{1} \mathrm{H} \mathrm{NMR}\left(400 \mathrm{MHz}, \mathrm{CDCl}_{3}\right): \delta(\mathrm{ppm}) 0.84-$ $0.99(\mathrm{~m}, 6 \mathrm{H}), 1.25-1.32(\mathrm{~m}, 2 \mathrm{H}), 1.63-1.72(\mathrm{~m}, 1 \mathrm{H}), 2.29(\mathrm{~s}, 3 \mathrm{H}), 3.14(\mathrm{~s}, 3 \mathrm{H}), 4.04-4.23(\mathrm{~m}, 1 \mathrm{H})$, 4.35-4.41 (m, 1H), $5.16(\mathrm{~d}, J 8.0 \mathrm{~Hz}, 1 \mathrm{H}), 5.36-5.39(\mathrm{~d}, \mathrm{br}, J 12.0 \mathrm{~Hz}, 2 \mathrm{H}), 7.17-7.76(\mathrm{~m}, 8 \mathrm{H}) .{ }^{13} \mathrm{C}$ NMR (100 MHz, $\left.\mathrm{CDCl}_{3}\right): \delta$ (ppm) 22.05, 22.92, 33.10, 41.56, 46.64, 47.58, 61.83, 67.40, 126.60, 
128.20, 128.40, 128.80, 141.00, 143.60, 154.00, 156.06. MS: Calc. for $\mathrm{C}_{23} \mathrm{H}_{28} \mathrm{~N}_{2} \mathrm{O}_{4}: \mathrm{m} / z$ 419.2000 $\left(\mathrm{M}^{+}+\mathrm{Na}\right)$, found: 419.2040 .

$\mathrm{N}^{\alpha}$-Fmoc-L-Trp-N(OCH $) \mathrm{CH}_{3}$ (2e). (9H-Fluoren-9-yl)methyl-3-(1 H-indol-3-yl)-1-(methoxy (methyl)amino)-1-oxopropan-2-ylcarbamate. Yield 87\%, gum. $\mathrm{R}_{f}$ (ethyl acetate/n- hexane 2:8) $=0.62, \mathrm{IR}\left(\mathrm{KBr}, \mathrm{cm}^{-1}\right): 1608 ;{ }^{1} \mathrm{H} \mathrm{NMR}\left(400 \mathrm{MHz}, \mathrm{CDCl}_{3}\right): \delta(\mathrm{ppm}) 2.75(\mathrm{~s}, 3 \mathrm{H}), 3.00(\mathrm{~m}, 2 \mathrm{H})$, $3.35(\mathrm{~s}, 3 \mathrm{H}), 4.48(\mathrm{~m}, 1 \mathrm{H}), 4.72-4.74(\mathrm{~d}, J 8.0 \mathrm{~Hz}, 2 \mathrm{H}), 4.90-4.91(\mathrm{t}, J 4.0 \mathrm{~Hz}, 1 \mathrm{H}), 6.74(\mathrm{~s}, 1 \mathrm{H})$, $7.18(\mathrm{~s}, 4 \mathrm{H}), 7.28-7.84(\mathrm{~m}, 8 \mathrm{H}), 5.00(\mathrm{~d}, \mathrm{br}, J 8.0 \mathrm{~Hz}, 1 \mathrm{H}),, 5.45(\mathrm{~d}, \mathrm{br}, J 8.0 \mathrm{~Hz}, 1 \mathrm{H}) .{ }^{13} \mathrm{C} \mathrm{NMR}$ (100 MHz, $\left.\mathrm{CDCl}_{3}\right): \delta$ (ppm) 31.05, 33.26, 47.10, 50.18, 61.84, 67.00, 110.84, 111.10, 119.00, $120.26,122.00,122.70,126.65,127.00,128.20,128.40,128.80,136.56,141.00,143.50,154.58$, 156.00. MS: Calc. for $\mathrm{C}_{28} \mathrm{H}_{27} \mathrm{~N}_{3} \mathrm{O}_{4}: m / z 492.2000\left(\mathrm{M}^{+}+\mathrm{Na}\right)$, found: 492.2002 .

$\mathrm{N}^{\alpha}$-Fmoc-L-Pro-N(OCH$) \mathrm{CH}_{3} \quad(2 \mathrm{f}) . \quad(\mathrm{S})-1-(9 H$-Fluoren-9-yl)methyl-2-(methoxymethyl) carbamoylpyrrolidine-1-carboxylate. Yield 90\%, gum. $\mathrm{R}_{f}$ (ethyl acetate/ $n$ - hexane 2:8): 0.65, IR (KBr, cm$\left.{ }^{-1}\right): 1667 ;{ }^{1} \mathrm{H}$ NMR (400 MHz, $\left.\mathrm{CDCl}_{3}\right): \delta(\mathrm{ppm}) 1.46(\mathrm{~m}, 2 \mathrm{H}) 1.74(\mathrm{~m}, 2 \mathrm{H}), 2.59(\mathrm{~s}, 3 \mathrm{H})$, $3.20(\mathrm{~m}, 2 \mathrm{H}), 3.45(\mathrm{~s}, 3 \mathrm{H}), 4.28-4.30(\mathrm{t}, J 4.0 \mathrm{~Hz}, 1 \mathrm{H}), 4.40-4.42(\mathrm{t}, J 4.0 \mathrm{~Hz}, 1 \mathrm{H}), 4.66-4.70$ (d, $J$ $8.0 \mathrm{~Hz}, 2 \mathrm{H}), 7.28-7.84(\mathrm{~m}, 8 \mathrm{H}) .{ }^{13} \mathrm{C} \mathrm{NMR}\left(100 \mathrm{MHz}, \mathrm{CDCl}_{3}\right): \delta$ (ppm) 22.00, 29.22, 32.94, 47.00, 47.35, 56.00, 61.55, 67.26, 126.80, 128.20, 128.40, 128.80, 141.00, 143.60, 154.50, 156.80. MS: Calc. for $\mathrm{C}_{22} \mathrm{H}_{24} \mathrm{~N}_{2} \mathrm{O}_{4}: m / z 403.1700\left(\mathrm{M}^{+}+\mathrm{Na}\right)$, found: 403.1736 .

$\mathbf{N}^{\alpha}$-Cbz-Ala-N $\left(\mathrm{OCH}_{3}\right) \mathrm{CH}_{3}$ (2g). (S)-Benzyl 1-(Methoxy(methyl)amino)-1-oxopropan-2ylcarbamate. Yield $86 \%, \mathrm{mp} 110{ }^{\circ} \mathrm{C}$. $\mathrm{R}_{f}$ (ethyl acetate/n- hexane 2:8) $=0.32, \mathrm{IR}\left(\mathrm{KBr}, \mathrm{cm}^{-1}\right)$ : 1650; ${ }^{1} \mathrm{H}$ NMR (400 MHz, $\left.\mathrm{CDCl}_{3}\right): \delta(\mathrm{ppm}) 1.33-1.35$ (d, $\left.J 8.0 \mathrm{~Hz}, 3 \mathrm{H}\right), 3.21(\mathrm{~s}, 3 \mathrm{H}), 3.77$ (s, $3 \mathrm{H}), 5.05-5.14(\mathrm{~m}, 1 \mathrm{H}), 5.30(\mathrm{~s}, 2 \mathrm{H}), 5.52-5.54(\mathrm{~d}, \mathrm{br}, J 8.0 \mathrm{~Hz}, 1 \mathrm{H}), 7.29-7.35(\mathrm{~m}, 5 \mathrm{H}) .{ }^{13} \mathrm{C} \mathrm{NMR}$ (100 MHz, $\left.\mathrm{CDCl}_{3}\right): \delta$ (ppm) 18.59, 32.14, 47.09, 61.57, 66.69, 127.96, 128.04, 128.45, 136.39, 154.55, 156.67. MS: Calc. for $\mathrm{C}_{13} \mathrm{H}_{18} \mathrm{~N}_{2} \mathrm{O}_{4}: m / z 289.1164\left(\mathrm{M}^{+}+\mathrm{Na}\right)$, found: 289.1166 .

$\mathrm{N}^{\alpha}$-Cbz-Ser-N $\left(\mathrm{OCH}_{3}\right) \mathrm{CH}_{3} \quad(2 \mathrm{~h}) . \quad(S)$-benzyl 3-Hydroxy-1-(methoxy(methyl)amino)-1oxopropan-2-ylcarbamate. Yield $80 \%$, gum. $\mathrm{R}_{f}($ ethyl acetate $/ \mathrm{n}$ - hexane $2: 8)=0.44$, IR $(\mathrm{KBr}$, $\left.\mathrm{cm}^{-1}\right)$ : 1673; ${ }^{1} \mathrm{H}$ NMR (400 MHz, $\left.\mathrm{CDCl}_{3}\right): \delta(\mathrm{ppm}) 1.98(\mathrm{~s}, 1 \mathrm{H}), 2.58(\mathrm{~s}, 3 \mathrm{H}), 3.16(\mathrm{~s}, 3 \mathrm{H}), 3.49-$ $3.79(\mathrm{~m}, 2 \mathrm{H}), 4.10-4.20(\mathrm{~m}, 1 \mathrm{H}), 5.10(\mathrm{~s}, 2 \mathrm{H}), 5.98-6.0(\mathrm{~d}, J 8.0 \mathrm{~Hz}, 1 \mathrm{H}), 7.24-7.32(\mathrm{~s}, 5 \mathrm{H}) .{ }^{13} \mathrm{C}$ NMR (100 MHz, $\left.\mathrm{CDCl}_{3}\right): \delta$ (ppm) 30.15, 52.96, 61.37, 62.66, 64.24, 127.84, 127.91, 128.25, 136.13, 154.69, 155.82. MS: Calc. for $\mathrm{C}_{13} \mathrm{H}_{18} \mathrm{~N}_{2} \mathrm{O}_{5}: \mathrm{m} / z$ 305.1216 $\left(\mathrm{M}^{+}+\mathrm{Na}\right)$, found: 305.1220 .

$\mathrm{N}^{\alpha}$-Cbz-Gly-N(OCH$) \mathrm{CH}_{3}$ (2i). Benzyl 2-(methoxy(methyl)amino)-2-oxoethylcarbamate. Yield 88\%, gum. $\mathrm{R}_{f}$ (ethyl acetate $/ n$ - hexane $\left.2: 8\right)=0.22$, IR $\left(\mathrm{KBr}, \mathrm{cm}^{-1}\right): 1630 ;{ }^{1} \mathrm{H}$ NMR (400 $\left.\mathrm{MHz}, \mathrm{CDCl}_{3}\right): \delta(\mathrm{ppm}) 2.5(\mathrm{~s}, 3 \mathrm{H}), 3.28(\mathrm{~s}, 3 \mathrm{H}), 3.65(\mathrm{~s}, 2 \mathrm{H}), 5.10(\mathrm{~d}, \mathrm{br}, J 4.0 \mathrm{~Hz}, 1 \mathrm{H}), 5.37$ (s, 2H), $7.18(\mathrm{~s}, 5 \mathrm{H}) .{ }^{13} \mathrm{C} \mathrm{NMR}\left(100 \mathrm{MHz}, \mathrm{CDCl}_{3}\right): \delta(\mathrm{ppm}) 31.2,38.0,62.5,66.4,127.2,127.8,128.2$, 141.0, 156.4, 164.3. MS: Calc. for $\mathrm{C}_{12} \mathrm{H}_{16} \mathrm{~N}_{2} \mathrm{O}_{4}: m / z 275.1100\left(\mathrm{M}^{+}+\mathrm{Na}\right)$, found: 275.1096 .

$\mathrm{N}^{\alpha}$-Boc-Phe-N(OCH$)_{3} \mathrm{CH}_{3}$ (2j). (S)-tert-Butyl 1-(methoxy(methyl)amino)-1-oxo-3-phenylpropan-2-ylcarbamate. Yield $90 \%, \mathrm{mp} 106{ }^{\circ} \mathrm{C}, \mathrm{R}_{f}($ ethyl acetate $/ n$ - hexane $2: 8)=0.29$, IR $(\mathrm{KBr}$, $\mathrm{cm}^{-1}$ ): 1648; ${ }^{1} \mathrm{H}$ NMR $\left(400 \mathrm{MHz}, \mathrm{CDCl}_{3}\right): \delta(\mathrm{ppm}) 1.37(\mathrm{~s}, 9 \mathrm{H}), 2.70(\mathrm{~s}, 3 \mathrm{H}), 3.15-3.17$ (d, $J 8.0$ $\mathrm{Hz}, 2 \mathrm{H}), 3.36(\mathrm{~s}, 3 \mathrm{H}), 4.79-4.80(\mathrm{t}, J 4.0 \mathrm{~Hz}, 1 \mathrm{H}), 5.24$ (d, br, J 8.0 Hz, 1H), 7.12-7.24 (m, 5H). ${ }^{13} \mathrm{C} \mathrm{NMR}\left(100 \mathrm{MHz}, \mathrm{CDCl}_{3}\right): \delta$ (ppm) 28.18, 31.94, 38.41, 51.44, 61.95, 77.31, 126.62, 128.27, 
129.23, 136.50, 155.07, 157.10. MS: Calc. for $\mathrm{C}_{16} \mathrm{H}_{24} \mathrm{~N}_{2} \mathrm{O}_{4}: m / z 331.1700\left(\mathrm{M}^{+}+\mathrm{Na}\right)$, found: 331.1710 .

$\mathrm{N}^{\alpha}$-Boc-Met-N(OCH$) \mathrm{CH}_{3}$ (2k). (S)-tert-Butyl 1-(methoxy(methyl)amino)-4-(methylthio)-1oxobutan-2-ylcarbamate. Yield $81 \%$, gum. $\mathrm{R}_{f}($ ethyl acetate $/ \mathrm{n}$ - hexane $2: 8)=0.36$, IR $\left(\mathrm{KBr}, \mathrm{cm}^{-}\right.$ $\left.{ }^{1}\right): 1653 ;{ }^{1} \mathrm{H}$ NMR $\left(400 \mathrm{MHz}, \mathrm{CDCl}_{3}\right): \delta(\mathrm{ppm}) 1.44(\mathrm{~s}, 9 \mathrm{H}), 2.09(\mathrm{~s}, 3 \mathrm{H}), 2.51-2.61(\mathrm{~m}, 2 \mathrm{H}), 2.90-$ $2.93(\mathrm{t}, J 4.0 \mathrm{~Hz}, 2 \mathrm{H}), 3.21(\mathrm{~s}, 3 \mathrm{H}), 3.78(\mathrm{~s}, 3 \mathrm{H}), 4.21-4.23(\mathrm{t}, J 4.0 \mathrm{~Hz}, 1 \mathrm{H}), 5.25-5.27$ (d, br, $J$ $8.0 \mathrm{~Hz}, 1 \mathrm{H}) .{ }^{13} \mathrm{C} \mathrm{NMR}\left(100 \mathrm{MHz}, \mathrm{CDCl}_{3}\right): \delta(\mathrm{ppm}) 15.25,27.59,30.04,31.42,32.07,49.72,61.54$, 79.54, 155.43, 172.48. MS: Calc. for $\mathrm{C}_{12} \mathrm{H}_{24} \mathrm{~N}_{2} \mathrm{O}_{4} \mathrm{~S} m / z: 315.1500\left(\mathrm{M}^{+}+\mathrm{Na}\right)$, found: 315.1550 .

$\mathrm{N}^{\alpha}$-Boc-Ile-N(OCH$)_{3} \mathrm{CH}_{3}$ (2l). tert-Butyl (2S,3S)-1-(methoxy(methyl)amino)-3-methyl-1oxopentan-2-ylcarbamate. Yield $80 \%$, gum. $\mathrm{R}_{f}$ (ethyl acetate $/ n$ - hexane $\left.2: 8\right)=0.35$, IR $(\mathrm{KBr}$, $\mathrm{cm}^{-1}$ ):1622; ${ }^{1} \mathrm{H}$ NMR (400 MHz, $\left.\mathrm{CDCl}_{3}\right): \delta(\mathrm{ppm})$ 0.94-.95 (t, $\left.J 4.0 \mathrm{~Hz}, 3 \mathrm{H}\right), 1.15-1.17$ (d, $J 8.0$ $\mathrm{Hz}, 3 \mathrm{H}), 1.29$ (m, 2H), 1.40 (s, 9H), $2.55(\mathrm{~m}, 1 \mathrm{H}), 2.74$ (s, 3H), 3.45 (s, 3H), 4.48-4.50 (d, J 8.0 $\mathrm{Hz}, 1 \mathrm{H}), 5.31$ (d, br, $J 8.0 \mathrm{~Hz}, 1 \mathrm{H}) .{ }^{13} \mathrm{C} \mathrm{NMR}\left(100 \mathrm{MHz}, \mathrm{CDCl}_{3}\right): \delta$ (ppm) 10.85, 14.70, 24.75, 28.50, 33.26, 37.48, 51.55, 61.85, 79.00, 154.00, 156.00. MS: Calc. for $\mathrm{C}_{13} \mathrm{H}_{26} \mathrm{~N}_{2} \mathrm{O}_{4} \mathrm{~m} / z 297.1900$ $\left(\mathrm{M}^{+}+\mathrm{Na}\right)$, found: 297.1942 .

$\mathrm{N}^{\alpha}$-Boc-Leu-N( $\left.\mathrm{OCH}_{3}\right) \mathrm{CH}_{3} \quad$ (2m). (S)-tert-Butyl 1-(methoxy(methyl)amino)-4-methyl-1oxopentan-2-ylcarbamate. Yield $86 \%$, gum, $\mathrm{R}_{f}$ (ethyl acetate/ $n$-hexane $\left.2: 8\right)=0.46$, IR $(\mathrm{KBr}$, $\mathrm{cm}^{-1}$ ): 1640; ${ }^{1} \mathrm{H} \mathrm{NMR}\left(400 \mathrm{MHz}, \mathrm{CDCl}_{3}\right): \delta(\mathrm{ppm})$ 0.98-1.00 (d, $\left.J 8.0 \mathrm{~Hz}, 6 \mathrm{H}\right), 1.35$ (s, 9H), 1.72 $(\mathrm{m}, 2 \mathrm{H}), 1.80(\mathrm{~m}, 1 \mathrm{H}), 2.68(\mathrm{~s}, 3 \mathrm{H}), 3.45(\mathrm{~s}, 3 \mathrm{H}), 4.64-4.67(\mathrm{t}, J 4.0 \mathrm{~Hz}, 1 \mathrm{H}), 5.18(\mathrm{~d}, \mathrm{br}, J 8.0 \mathrm{~Hz}$, 1H). $\left.{ }^{13} \mathrm{C} \mathrm{NMR} \mathrm{(100} \mathrm{MHz,} \mathrm{CDCl}_{3}\right): \delta$ (ppm) 22.00, 22.35, 28.52, 31.90, 40.74, 46.57, 61.46, 79.00, 154.40, 156.50. MS: Calc. for $\mathrm{C}_{13} \mathrm{H}_{26} \mathrm{~N}_{2} \mathrm{O}_{4}: m / z 297.1900\left(\mathrm{M}^{+}+\mathrm{Na}\right)$, found: 297.1890 .

\section{$\mathrm{N}^{\alpha}$-Fmoc-L-Gly-Pro-N $\left(\mathrm{OCH}_{3}\right) \mathrm{CH}_{3} \quad(4 \mathrm{n}) . \quad(R)-(9 H$-Fluoren-9-yl)methyl-2-(2-} (methoxy(methyl)carbamoyl)pyrrolidin-1-yl)-2-oxoethylcarbamate. Yield $90 \%$, gum. $\mathrm{R}_{f}$ (ethyl acetate/ $n$-hexane 2:8) $=0.26, \mathrm{IR}\left(\mathrm{KBr}, \mathrm{cm}^{-1}\right): 1655 ;{ }^{1} \mathrm{H} \mathrm{NMR}\left(400 \mathrm{MHz}, \mathrm{CDCl}_{3}\right): \delta(\mathrm{ppm})$ 1.85-1.86 (m, 2H), $2.05(\mathrm{~m}, 2 \mathrm{H}), 2.70(\mathrm{~s}, 3 \mathrm{H}), 3.29(\mathrm{~s}, 3 \mathrm{H}), 3.40-3.42(\mathrm{t}, J 4.0 \mathrm{~Hz}, 2 \mathrm{H}), 3.78(\mathrm{~s}$, $2 \mathrm{H}), 4.37-4.39(\mathrm{t}, J 4.0 \mathrm{~Hz}, 1 \mathrm{H}), 4.48-4.50(\mathrm{t}, J 4.0 \mathrm{~Hz}, 1 \mathrm{H}), 4.58-4.60$ (d, J 8.0 Hz, 2H), 5.05 (d, br, $J 4.0 \mathrm{~Hz}, 1 \mathrm{H}) 7.25-7.84(\mathrm{~m}, 8 \mathrm{H}) .{ }^{13} \mathrm{C}$ NMR (100 MHz, $\left.\mathrm{CDCl}_{3}\right): \delta$ (ppm) 22.25, 29.74, 33.44, $42.00,44.98,46.80,54.55,61.90,67.25,126.80,128.20,128.40,128.80,141.00,143.55,154.00$, 156.25, 167.80. MS: Calc. for $\mathrm{C}_{24} \mathrm{H}_{27} \mathrm{~N}_{3} \mathrm{O}_{5}: m / z 447.1872\left(\mathrm{M}^{+}+\mathrm{Na}\right)$, found: 447.1868 .

$\mathrm{N}^{\alpha}$-Fmoc-L-Arg-Gly-N(OCH$) \mathrm{CH}_{3} \quad$ (40). (S)-(9H-fluoren-9-yl)methyl 5-guanidino-1-(2 (methoxy(methyl)amino)-2-oxoethylamino)-1-oxopentan-2-ylcarbamate. Yield 88\%, gum. $\mathrm{R}_{f}$ (ethyl acetate $/ n$-hexane 2:8) $=0.38, \mathrm{IR}\left(\mathrm{KBr}, \mathrm{cm}^{-1}\right): 1665 ;{ }^{1} \mathrm{H} \mathrm{NMR}\left(400 \mathrm{MHz}, \mathrm{CDCl}_{3}\right): \delta(\mathrm{ppm})$ $1.50(\mathrm{~m}, 2 \mathrm{H}), 1.76(\mathrm{~m}, 2 \mathrm{H}), 2.00(\mathrm{~s}, 3 \mathrm{H}), 2.58-2.59(\mathrm{t}, J 4.0 \mathrm{~Hz}, 2 \mathrm{H}), 2.70(\mathrm{~s}, 3 \mathrm{H}), 3.00(\mathrm{~s}, 1 \mathrm{H})$, $3.24(\mathrm{~s}, 2 \mathrm{H}), 4.05(\mathrm{~s}, 1 \mathrm{H}), 4.36(\mathrm{~s}, 2 \mathrm{H}), 4.45(\mathrm{~m}, 1 \mathrm{H}), 4.58(\mathrm{~m}, 1 \mathrm{H}), 4.70-4.74(\mathrm{~d}, J 8.0 \mathrm{~Hz}, 2 \mathrm{H})$, $5.10(\mathrm{~d}, \mathrm{br}, J 8.0 \mathrm{~Hz}, 2 \mathrm{H}), 7.28-7.84(\mathrm{~m}, 8 \mathrm{H}) .{ }^{13} \mathrm{C} \mathrm{NMR}\left(100 \mathrm{MHz}, \mathrm{CDCl}_{3}\right): \delta(\mathrm{ppm}) 24.05,28.65$, $32.70,37.24,37.56,47.00,54.55,61.76,66.95,126.80,128.20,128.40,128.80,141.00,143.60$, 156.00, 158.50, 164.55, 171.00. MS: Calc. for $\mathrm{C}_{25} \mathrm{H}_{32} \mathrm{~N}_{6} \mathrm{O}_{5}: m / z 519.2434\left(\mathrm{M}^{+}+\mathrm{Na}\right)$, found: 519.2430 .

$\mathrm{N}^{\alpha}$-Fmoc-L-Arg-Ala-N(OCH$) \mathrm{CH}_{3}$ (4p). (9H-fluoren-9-yl)methyl (S)-5-guanidino-1-((R)-1(methoxy(methyl)amino)-1-oxopropan-2-ylamino)-1-oxopentan-2-ylcarbamate. Yield 84\%, 
gum. $\mathrm{R}_{f}$ (ethyl acetate/ $n$-hexane 2:8) $=0.3$, IR $\left(\mathrm{KBr}, \mathrm{cm}^{-1}\right): 1657 ;{ }^{1} \mathrm{H} \mathrm{NMR}\left(400 \mathrm{MHz}, \mathrm{CDCl}_{3}\right): \delta$ (ppm) $1.50(\mathrm{~m}, 2 \mathrm{H}), 1.76(\mathrm{~m}, 2 \mathrm{H}), 2.00(\mathrm{~s}, 3 \mathrm{H}), 2.58-2.59$ (t, J 4.0 Hz, 2H), $2.70(\mathrm{~s}, 3 \mathrm{H}), 3.00$ (s, $1 \mathrm{H}), 3.24(\mathrm{~s}, 2 \mathrm{H}), 4.05(\mathrm{~s}, 1 \mathrm{H}), 4.36(\mathrm{~s}, 2 \mathrm{H}), 4.45(\mathrm{~m}, 1 \mathrm{H}), 4.58(\mathrm{~m}, 1 \mathrm{H}), 4.70-4.74(\mathrm{~d}, J 8.0 \mathrm{~Hz}$, $2 \mathrm{H}), 5.10$ (d, br, $J 8.0 \mathrm{~Hz}, 2 \mathrm{H}), 7.28-7.84(\mathrm{~m}, 8 \mathrm{H}) .{ }^{13} \mathrm{C} \mathrm{NMR}\left(100 \mathrm{MHz}, \mathrm{CDCl}_{3}\right): \delta(\mathrm{ppm}) 24.05$, 28.65, 32.70, 37.24, 37.56, 47.00, 54.55, 61.76, 66.95, 126.80, 128.20, 128.40, 128.80, 141.00, 143.60, 156.00, 158.50, 164.55, 171.00. MS: Calc. for $\mathrm{C}_{26} \mathrm{H}_{34} \mathrm{~N}_{6} \mathrm{O}_{5}: m / z 533.2591\left(\mathrm{M}^{+}+\mathrm{Na}\right)$, found: 533.2589 .

\section{Biological Studies}

To evaluate the binding efficacy and inhibitory effects of synthesized compounds, three protein targets from three different organisms, namely Escherichia coli, Pseudomonas aeroginosa and Staphylococcus aureus, were selected for this study. The X-ray crystallographic structure of enoylACP reductase (1C14) Escherichia coli, X-ray crystallographic structure of LasR ligand binding domain bound to its natural ligand $n$-3-oxododecanoyl-L-homoserine lactone (2UV0) Pseudomonas aeroginosa and X-ray crystallographic structure of dehydrosqualene synthase (2ZCP) Staphylococcus aureus ${ }^{24-26}$ respectively were obtained from Protein Data Bank (PDB) ${ }^{23}$. The prepared targets were then used for molecular docking studies ${ }^{27}$ which will predict possible ligand interactions with the active site residues that can inhibit protein activity. To perform docking, FlexX module of LeadIT software was used. ${ }^{28}$ The LeadIT score obtained after the docking were considered to know the free binding energy $(\Delta \mathrm{G})$. Based on the very low binding energy and maximum possible intermolecular interactions, best lead compounds with strong binding efficacy were finalized. 2D structures of all the ligands were drawn in ChemDraw Ultra 8.0 and were exported as mol file for further processing in DS 3.5. The generated conformers were optimized using CHARMm force field ${ }^{29}$ and then minimized. Each selected conformers were then grouped into one library file and were subjected to docking. The FlexX docking score correlates with the binding affinity of the molecules with the target.

Antibacterial activity was screened by Agar well diffusion method ${ }^{30}$ against three pathogenic bacterial strains, Escherichia coli MTCC1692, Pseudomonas aeroginosa MTCC1688 and Staphylococcus aureus MTCC3160 (one gram +ve and two gram -ve). Preliminary screening was done to check antibacterial activities of synthesized compounds over Muller-Hinton agar plates. The inhibition zones obtained were measured in millimeters against the Streptomycin sulfate standard. Finally, the average values were considered for the ultimate antibacterial activity.

\section{Acknowledgements}

We thank the Principal and Director of Siddaganga Institute of Technology, Tumakuru, Karnataka, for the research facilities. One of the authors (HSL) is thankful to the Vision Group of Science and Technology (VGST), Department of Information Technology, Biotechnology and Science \& 
Technology, Government of Karnataka for providing funds to carry out the present research work by means of a sponsored project.

\section{References}

1. Katritzky, A.R.; Yang, H.; Zhang, S.; Wang, M. Arkivoc 2002, (xi), 39-44. http://dx.doi.org/10.3998/ark.5550190.p009.385

2. Liu, J.; Ikemoto, N.; Petrillo, D.; Armstrong, III J.D. Tetrahedron Lett. 2002, 43, 8223-8226. http://dx.doi.org/10.1016/S0040-4039(02)02031-2

3. Sureshbabu, V.V.; Hemantha, H. P.; Arkivoc 2008, (ii), 243-249.

4. Tunoori, A. R.; White, J. M.; Georg, G. I. Org. Lett. 2000, 2, 4091-4093. http://dx.doi.org/10.1021/o1000318w

5. Bailen, M.A.; Chinchilla, R.; Dodsworth, D.J.; Najera, C. Tetrahedron Lett. 2001, 42, 50135016. http://dx.doi.org/10.1016/S0040-4039(01)00923-6

6. Kim, J.G.; Jang, D.O. Bull. Korean Chem. Soc. 2010, 31, 171-173. http://dx.doi.org/10.5012/bkcs.2010.31.01.171

7. Krishnamoorthy, R.; Lam, S.Q.; Manley, C.M.; Herr, R.J. J. Org. Chem. 2010, 75, 12511258. http://dx.doi.org/10.1021/jo902647h

8. Luca, L.D.; Giacomelli, G.; Taddei, M. J. Org. Chem. 2001, 66, 2534 -2537. http://dx.doi.org/10.1021/jo015524b

9. Deagostino, A.; Larini, P.; Occhiato, E.G.; Pizzuto, L.; Prandi, C.; Venturello, P. J. Org. Chem. 2008, 73, 1941-1945. http://dx.doi.org/10.1021/jo7024898

10. Amit Kumar; Akula, H.K.; Lakshman, M.K. Eur. J. Org. Chem. 2010, 14, 2709- 2715. http://dx.doi.org/10.1002/ejoc.200901420

11. Sharnabai, K.M.; Nagendra, G.; Vishwanatha, T.M.; Sureshbabu, V.V. Tetrahedron Lett. 2013, 54, 478-482. http://dx.doi.org/10.1016/j.tetlet.2012.11.064

12. Tyrrell, E.; Brawn, P.; Carew, M.; Greenwood, I. Tetrahedron Lett. 2011, 52, 369-372. http://dx.doi.org/10.1016/j.tetlet.2010.10.169

13. Paul, R.; Anderson, G.W. J. Am. Chem. Soc. 1960, 82, 4596-4600. http://dx.doi.org/10.1021/ja01502a038

14. Wang, Z.Z.; Ma, Y.J. J. Chem. Pharm. Res. 2012, 4, 580-582.

15. Montalbetti, C.A.G.N., Falque, V. Tetrahedron. 2005, 61, 10827-10852. http://dx.doi.org/10.1016/j.tet.2005.08.031

16. Grzyb, J.A., Batey, A. Tetrahedron Lett. 2003, 44, 7485-7488. http://dx.doi.org/10.1016/j.tetlet.2003.08.026 
17. Vasantha, B.; Vishwanatha, T.M.; Sureshbabu, V.V. Protein Pept. Lett. 2011, 18, $1093-$ 1098.

http://dx.doi.org/10.2174/092986611797200922

18. Staab, H.A.; Bauer, H.; Schneider, K.M. in Azolides in Organic Synthesis and Biochemistry. Wiley-VCH: Germany, 1998, 282-283. http://dx.doi.org/10.1002/3527600833

19. Wahab, B.F.A.; Mohamed, H.A. Turk. J. Chem. 2012, 36, 805-826.

20. Katritzky, A.R.; Singh, S.K.; Cai, C.; Bobrov, S. J. Org. Chem. 2006, 71, 3364-3374. http://dx.doi.org/10.1021/jo052293q

21. Waszkowycz, B.; Clark, D.E.; Gancia, E. Wiley Interdisciplinary Reviews: Computational Molecular Science. 2011, 1, 229-259. http://dx.doi.org/10.1002/wcms.18

22. Dammalli, M.; Vivek, C.; Biradar, M.I.; Navya, N., Bhavya, S.G. Asian Pac. J. Trop. Diseases 2014, 4, 635-640. http://dx.doi.org/10.1016/S2222-1808(14)60694-0

23. Protein Data Bank. Nature New Biol. 1971, 233, 223.

24. Qiu, X.; Abdel-Meguid, S.S.; Janson, C.A.; Court, R.I.; Smyth, M.G.; Payne, D. J. Protein Sci. 1999, 8, 2529-2532. http://dx.doi.org/10.1110/ps.8.11.2529

25. Song, Y.; Liu, C.I.; Lin, F.Y.; Hwan No, J.; Hensler, M.; Liu, Y.L.; Jeng, W.Y.; Low,J.; Liu, G.Y.; Nizet, V.; Wang, A.H.J.; Oldfield, E. J. Med. Chem. 2009, 52, 3869-3880. http://dx.doi.org/10.1021/jm9001764

26. Schwefel, D.; Maierhofer, C.; Beck, J.G.; Seeberger, S.; Diederichs, K.; Moller, H.M.; Welte, W.; Wittmann, V. J. Am. Chem. Soc. 2010, 132, 8704-8719. http://dx.doi.org/10.1021/ja101646k

27. Jiang, X.R.; Wang, P.; Smith, C.L.; Zhu B.T. J. Med. Chem. 2013, 56, 2779-2790. http://dx.doi.org/10.1021/jm3013773

28. Nakao, T.; Banba, S.; Nomura, M.; Hirase, K. Insect Biochem. Mol. Biol. 2013, 43, 366-375. http://dx.doi.org/10.1016/j.ibmb.2013.02.002

29. Vivek, C.; Anubhav, K.; Mamatha, C.; Sindhu, G.; Gowrishankar, B.S. Adv. Virology 2015, 1-9.

30. Palaksha, M.; Ahmed, M.; Das, S. J. Nat. Sci., Biol.Med. 2010, 1, 12-15. http://dx.doi.org/10.4103/0976-9668.71666 